\title{
Exploring Decoy Effects on Computerized Task Preferences in Rhesus Monkeys (Macaca mulatta)
}

\author{
Audrey E. Parrish ${ }^{1 *}$, Elyce Afrifa², and Michael J. Beran ${ }^{3}$ \\ ${ }^{1}$ Psychology Department, The Citadel, Charleston, SC \\ ${ }^{2}$ Herbert H. Lehman High School, New York, NY \\ ${ }^{3}$ Psychology Department \& Language Research Center, Georgia State University, Atlanta, GA \\ *Corresponding author (Email: audrey.parrish1@gmail.com)
}

Citation - Parrish, A. E., Afrifa, E., \& Beran, M. J. (2018). Exploring decoy effects on computerized task preferences in rhesus monkeys (Macaca mulatta). Animal Behavior and Cognition, 5(2), 235-253. https://doi.org/10.26451/abc.05.02.06.2018

Note: This paper is the final report based on an accepted peer-reviewed preregistered submission that can be found here.

\begin{abstract}
The asymmetric dominance effect or decoy effect emerges when a third inferior option is introduced to a choice set. The decoy option, although typically not chosen, impacts relative preference for the original two options. This decisional bias stands in contrast with rational choice theory, which dictates that choice behavior should remain consistent for the original options with the addition of different alternatives to a choice set such as the decoy. In the current study, we assessed the decoy effect in rhesus monkeys using a computerized task battery that introduced two different computerized tasks, including a matching-to-sample task and a psychomotor task called PURSUIT. Decoy tasks were designed such that they were inferior versions of these original task options, requiring longer time to completion (via slowed cursor speeds) and subsequently reduced reinforcement rates. Monkeys learned to associate unique icons for each task (including for decoy tasks), and used these icons to select their preferred task from a choice set of two to three task options. Monkeys learned to perform all tasks, but did not show evidence of the decoy effect using this task preference paradigm. We discuss the role of initial task preference (and task biases), task type (symbolic vs. perceptual), and decoy effect sizes in light of these findings. We contrast the current results to previous findings of the decoy effect in rhesus monkeys using a perceptual paradigm as well as to other evidence of the decoy effect in non-primate animal species.
\end{abstract}

Keywords - Decoy effect, Asymmetric dominance effect, Decision-making, Rhesus monkeys

Choice behavior reveals a wealth of information regarding one's preferences, perceptions, thoughts, and ideas, and, of equal importance, the factors that impact decision-making. To this latter point, choice behavior does not occur in a vacuum. Rather, choices are largely context-dependent in terms of the environment in which decisions are made. One clear example of context-dependent choice behavior that is prevalent across a variety of domains and includes a growing number of species is the asymmetric dominance effect (ADE), also referred to as the decoy effect (for a review, see Huber, Payne, \& Puto, 2014). In the decoy effect, a decision-maker chooses among two options that differ on multiple dimensions and the relative preference between original options is altered following the introduction of a third weaker option (the decoy). In the original choice set, the first option (e.g., an expensive 5-star restaurant) dominates the second option (e.g., a less-expensive 3-star restaurant) on one dimension 
(quality) whereas the second option dominates the first option on a separate dimension (price). The decision-maker may experience indifference or a slight preference between these options if both dimensions (restaurant quality and monetary savings) are of equivalent value. A third, weaker, option known as the decoy is introduced to the choice set that is asymmetrically dominated by one of the original options (e.g., an even more expensive 4-star restaurant is weaker on both dimensions of price and quality relative to the 5-star restaurant). The decoy is typically rarely chosen as it is seen as inferior or even nonviable. However, it can serve to increase the decision maker's preference for the original, relatively superior option (e.g., the original 5-star restaurant).

According to rational choice theory, the inferior decoy option is not expected to play a part in choice behavior as options should be evaluated independently of one another, leading to stable preferences among options regardless of the introduction of additional alternatives (Luce, 1977). However, the decoy often results in a preference shift for the original options (away from indifference or an increase/decrease in the preference for one of the original options; Huber, Payne, \& Puto, 1982). The decoy effect has been studied extensively within behavioral economics, with a considerable amount of research concerning consumer decision-making scenarios among human adults (e.g., Doyle, O'Connor, Reynolds, \& Bottomley, 1999; Huber et al., 1982; Parducci, 1965; Pettibone \& Wedell, 2000; Wedell, 1991; Zhang \& Zhang, 2007), as well as mate preference (Sedikides, Ariely, \& Olsen, 1999) and political elections (Herne, 1997; Pan, O’Curry, \& Pitts, 1995).

The decoy effect also has been documented among a number of nonhuman animals, beginning with compelling work with starlings (Sturnus vulgaris: Bateson, 2002) and hummingbirds (Selasphorus rufus: Bateson, Healy, \& Hurly, 2002), and extending to honeybees and gray jays (Apis mellifera and Perisoreus canadensis: Shafir, Waite, \& Smith, 2002), cats (Felis catus: Scarpi, 2011), and primates (Cebus apella; Cohen \& Santos, 2017; Macaca mulatta: Parrish, Evans, \& Beran, 2015). For example, Shafir et al. (2002) documented preference shifts in a foraging task with honeybees and gray jays that were presented with binary (2-alternative) choices versus trinary (3-alternative) choice options that contained a decoy alternative. Bees, for instance, were presented with artificial flowers that varied on two dimensions: sucrose volume (i.e., preferred food type) and flower tube length (i.e., effort expenditure to obtain sucrose). The binary choice set presented a $40 \mathrm{~mm}$ tube $/ 2 \mu \mathrm{l}$ sucrose flower versus a $50 \mathrm{~mm}$ tube $/ 3$ $\mu 1$ sucrose flower. Half of the bees experienced decoy flowers that were dominated based on tube length (100 mm tube $/ 3 \mu \mathrm{l}$ decoy flowers were dominated by the original $50 \mathrm{~mm}$ tube/ $3 \mu \mathrm{l}$ flower) and half of the bees experienced decoy flowers that were dominated based on sucrose volume $(40 \mathrm{~mm}$ tube/1 $\mu$ l decoy flowers were dominated by the original $40 \mathrm{~mm}$ tube/ $2 \mu \mathrm{l}$ flower). The bees' relative preference between the two original flowers was altered such that there was a greater proportion choice behavior for the option that was most similar to the decoy (e.g., the original 50/3 flower was chosen at a higher rate than the original 40/2 flower when the 100/3 decoy flower was present). Similar effects were documented for jays in which preference for a target option was increased following the inclusion of a decoy that was dominated on one of two dimensions. Thus, the bees' and jays' choice options were not valued independently of the alternative choices; decisions were seemingly made upon a comparative basis in which both preferred options and less-preferred alternatives (i.e., the decoy) shaped the insects' and birds' choice behavior. Different tasks have been developed in the comparative literature that vary how choices are presented, providing substance to the argument that decoy effects are far-reaching, emerging across a variety of contexts, and among a diverse group of species. Such comparative evidence indicates that context effects have been at work in choice behavior well before the evolution of modern humans and likely plays a widespread role in decision-making.

In our previous work investigating choice behavior among primates, we presented rhesus macaques (Macaca mulatta) with a computerized perceptual discrimination task to investigate the decoy effect as the first study to assess this phenomenon in a nonhuman primate species (Parrish et al., 2015). We were interested in whether decoy effects might emerge using a relatively simple perceptual choice task modified from the human literature (Trueblood, Brown, Heathcote, \& Busemeyer, 2013), which would provide further support for the idea that these decisional biases are early emerging, quickly generated, and widespread across contexts. When presented with a size-judgment task, monkeys 
successfully discriminated two rectangles that varied in size and orientation (horizontally or vertically oriented). Monkeys performed well in the baseline binary task, successfully choosing the larger of two rectangles across a variety of difficulty levels in terms of size discrepancy. When introduced with a third rectangle smaller in size than both rectangles, monkeys rarely chose this inferior 'decoy' option. However, their performance in selecting the largest rectangle from the remaining two choices during these probe trials was increased relative to baseline when the decoy was oriented in the same direction as the largest rectangle (e.g., the decoy and largest rectangle were longer in the horizontal plane, increasing its likelihood of selection). Alternatively, if the decoy rectangle's orientation was incongruent with the truly largest rectangle, performance suffered (e.g., the decoy and second-to-largest rectangle were longer in the horizontal plane, increasing likelihood of selection of the latter). These comparative results from Parrish et al. (2015) indicated that rhesus monkeys responded similarly to decoy stimuli in a perceptual discrimination task as human adults (Trueblood et al., 2013). The use of this version of a perceptual decoy paradigm also highlighted that context effects of this nature are not exclusive to complex decisionmaking, such as consumer choice behavior or political elections, or to inherently valuable and prepotent stimuli common for comparative tasks, such as choices among appetitive food options. Rather, such decoy effects emerge within a wide range of contexts and across a variety of species.

In the current study, we extended these findings to a different kind of assessment of the decoy effect using choice options and decoys that were not perceptual or appetitive. Rather, we gave monkeys choices between icons for computerized tasks that they could perform with decoy task icons sometimes presented to determine how inferior alternatives affected the relative preference among non-decoy tasks. In this paradigm, the tasks were represented by visual icons, which the monkeys could select to indicate their task preference. All tasks varied on multiple dimensions including the effort to obtain reward outcomes. Importantly, the emergence of the decoy effect among the same species across paradigms (i.e., from a basic, early-emerging perceptual level to a more complex and multi-faceted decisional task) would shed light on the degree to which decisional biases impact choice behavior in a variety of settings and across a range of cognitive complexity.

This new task also assesses whether representational or symbolic decoy effects occur in other species. In fact, many examples of human decoy effects occur among choice options that contain symbolic representations rather than being choices directly comparable in terms of perceptual features (e.g., abstract policy decisions when comparing potential political candidates or comparisons of price and quality such as when one compares potential car purchases). Thus, for humans, decoy effects occur "early" in decision-making, at the level of perceptual experience, but also "later" in terms of evaluating options on the basis of non-perceptual factors (see Trueblood et al., 2013). Such evidence in monkeys would indicate that decisional biases for representational stimuli perhaps are not uniquely human and may be present among other species.

In the current task, monkeys were presented with several task types (each represented by a unique icon) that varied in effort expenditure to complete a trial. After learning to associate each icon with a specific task and its parameters, and ensuring that monkeys showed reliable preferences among those tasks, we introduced decoy icons. The tasks associated with these decoy icons were objectively inferior to the original tasks, requiring a longer time to complete and reduced reinforcement rate. Using this choice paradigm, we measured preference behavior and subsequent shifts in preferences by presenting monkeys with the option of completing one of two or three tasks (binary choice set vs. trinary choice set). We predicted that the presence of the decoy task option in the trinary choice set would impact choice behavior, such that an asymmetrically dominated option (i.e., one that is rarely selected itself) would lead to overall higher preferences for the similar non-decoy task.

We established a context for potential decoy effects during computerized tasks by making use of the SELECT experimental procedure in which monkeys have learned to use a joystick to control a cursor on the computer screen. Washburn, Hopkins and Rumbaugh (1991) originally described the SELECT procedure. They presented rhesus macaques with a choice among different icons on a computer screen, and each icon represented a different experimental task. They varied the parameters to generate objectively easier or more difficult tasks to see if monkeys would learn to use the icons to show 
preferences for tasks. Monkeys learned these icons, and established predictable, reliable, and stable choice patterns among tasks. Washburn et al. (1991) also found that the use of the SELECT framework for testing monkeys produced other interesting outcomes. For example, monkeys performed better on some tasks when they had chosen them (i.e., choice trials) than when the same tasks were simply assigned to them (i.e., forced trials). Later work replicated and extended this result with capuchin monkeys (Beran, Klein, Evans, Antworth, \& Chan, 2007; Perdue, Evans, Washburn, Rumbaugh, \& Beran, 2014).

This SELECT paradigm lends itself to assessing potential decoy effects in terms of choice behavior and potential preference shifts. We first trained monkeys to associate four icons with two versions of two different tasks. One task was a psychomotor task called PURSUIT that required the monkeys to track a moving target with their cursor, and then maintain contact with that moving target. The other task was an identity matching-to-sample (MTS) task that required selecting a visual stimulus from two options that matched a presented sample image. These two tasks are well-known games for these monkeys, but we also manipulated each to attempt to generate a favored version and an objectively inferior or dominated (decoy) version in terms of effort. For the PURSUIT task, the decoy version increased the duration of required continued contact with the moving target. For the MTS task, we greatly slowed the speed of the cursor in the decoy version, so that the monkeys had to work longer to make the same matching response. Thus, the decoy version of each task required a greater degree of effort to complete a trial, making each decoy objectively less rewarding.

First, monkeys gained experience with each task (normal version and decoy version) and its SELECT icon by being presented with only one choice icon, and then one trial of the task associated with that icon. These icons were interspersed and randomly presented across the first four trials of each block of 10 training trials. Thus, all icons and their associated tasks were experienced to an equivalent degree. Then, in the last six trials of each 10-trial block in training, monkeys were given the MTS and PURSUIT icons to choose between. Monkeys remained in the training phase until they showed choices that were not extreme for one or the other of these tasks. Finally, in the test phase, MTS and PURSUIT were presented as the two choices, as were each of those tasks with their respective decoy versions. The crucial test trial types were those in which MTS and PURSUIT were options along with one of the two decoy icons. In those trials, the prediction was that monkeys would increase their relative choice of the PURSUIT task to the MTS task when the PURSUIT decoy was present, but increase their relative choices of the MTS task when the MTS decoy was present. This pattern would reflect the decoy effect, and would highlight that this effect occurs in nonhuman species even in contexts in which the decoy stimuli are symbolic of the subsequent tasks that the monkeys still had to perform.

In addition to the manipulations that would let us assess decoy effects, our use of the SELECT task allowed us to further study these monkeys' preferences for tasks that vary in the dimensions of effort and outcome. Although not a central feature of the design, such data also are informative and will reflect another benefit of the SELECT task in assessing monkey preferences.

\section{Experiment 1}

\section{Method}

Participants. We tested seven adult male rhesus monkeys between the ages of 13 and 34 years. All monkeys previously had been trained to use the computerized apparatus and had experience with the MTS and PURSUIT tasks (Evans, Beran, Chan, Klein, \& Menzel, 2008; Richardson, Washburn, Hopkins, Savage-Rumbaugh, \& Rumbaugh, 1990), including in tests in which the SELECT procedure was used (Beran et al., 2007; Perdue et al., 2014). Monkeys had access to the computer in test sessions that typically ranged from $4-8 \mathrm{hrs}$ during which time the software program was available onscreen. They could engage with the computerized apparatus as they chose, and they otherwise rested or engaged with other enrichment in their enclosure throughout these sessions. Monkeys always had access to water, and were given a daily meal each afternoon independent of whether they worked on the computer task. Monkeys also spent time each week with outdoor access. 
Apparatus. The monkeys were tested using the Language Research Center's Computerized Test System, which consisted of a personal computer, digital joystick, color monitor, and pellet dispenser (Evans et al., 2008; Richardson et al., 1990). Monkeys manipulated the joystick with their hands so as to move a cursor onscreen during tasks. Monkeys earned $94 \mathrm{mg}$ banana-flavored chow pellets as food rewards (Bio-Serv, Frenchtown, NJ), and those pellets were delivered via a pellet dispenser that was connected to the computer. The software for the tasks was written in Visual Basic 6.0.

Design and procedure. Two computerized tasks were used for this experiment. These included the MTS and PURSUIT tasks described below. A decoy version of each task also was created. Tasks were then presented in binary and trinary choices using the SELECT paradigm, in which monkeys chose between two or three icons associated with each task. Selection of a task icon led to the presentation of the associated task.

PURSUIT task. In this task, monkeys moved the cursor onscreen using their joystick to capture a moving target (see Figure 1). At the start of each trial, the cursor appeared at a random position on the monitor screen, and a green circle (called the target) also appeared randomly on the screen. Deflection of the joystick in any direction by the monkey led to simultaneous movement of the cursor and the target. When the cursor entered the target, the target changed color as long as the cursor remained inside of it. When the target reached an edge of the screen, it reversed direction in such a way that it appeared to bounce off the edge of the screen. If the monkey stopped moving the cursor when the cursor was not in the target, the target also stopped moving. But, if a monkey stopped moving the cursor when the cursor was inside of the target, the target would keep moving, and the cursor would lose "contact" with it. After two minutes, if the monkey did not successfully track the target for the full duration, the trial ended.

For the non-decoy version of the task, the monkey initially had to maintain contact of the cursor inside the target for $1 \mathrm{~s}$ to generate a 1-pellet food reward, a melodic tone, and then return to the SELECT screen. For the decoy version, the monkey had to maintain contact inside the target for $5 \mathrm{~s}$, and received a 1-pellet reward and the melodic tone. If necessary, the parameter of contact duration was adjusted at the end of sessions (see below for details).

MTS task. In this task, monkeys moved the cursor to a sample clip-art stimulus presented in the center of the screen, at which time two comparison clip-art stimuli were presented in the top left and top right corners of the screen. The sample also remained present onscreen (see Figure 1). One of the two comparison stimuli was identical to the sample and was the correct choice. If the correct stimulus was contacted with the cursor, the monkey received a single food pellet and melodic auditory feedback. An incorrect response (i.e., contact with the non-matching stimulus) resulted in a time-out penalty of $5 \mathrm{~s}$ and buzzing auditory feedback. After 2 min, if the monkey did not select a match option, the trial ended.

In the non-decoy version of the MTS task, initial movement of the cursor to one of the two matching options took approximately $1 \mathrm{~s}$ of continuous joystick deflection to complete, whereas in the decoy version of the task, it took a minimum of $5 \mathrm{~s}$ to make the same response. If necessary, this parameter of cursor speed was adjusted (see below for details).

SELECT task training and testing. The task was a modified version of the SELECT task that had previously been presented to monkeys at the LRC (e.g., Beran et al., 2007; Washburn et al., 1991). In this modified version, four arbitrarily designated icons were used to represent the four tasks, and they could appear in any of four locations to the left, right, top or bottom center of the screen (see Figure 1). These icons were novel to the current task; thus, the monkeys could not have memorized the presented task icons from previous studies. Each session occurred in four phases:

Training phase. This phase consisted of blocks of 10 trials. Within each block, the first four trials consisted of presenting only one icon to the monkey. Within these four randomly arranged trials, one trial was a forced MTS trial, one trial was a forced PURSUIT trial, one trial was a forced decoy-MTS trial, and one trial was a forced decoy-PURSUIT trial. These trials were designed to train the monkeys to associate each icon with each specific task. For the remaining six trials in each block, the monkey was presented with two icons, and the monkey had to choose one of those icons to then be given the associated task. This comparison was between the MTS vs. PURSUIT icons. The resulting data from those trials constituted the baseline preference for each monkey for each of these two tasks. 
Parrish et al. 240

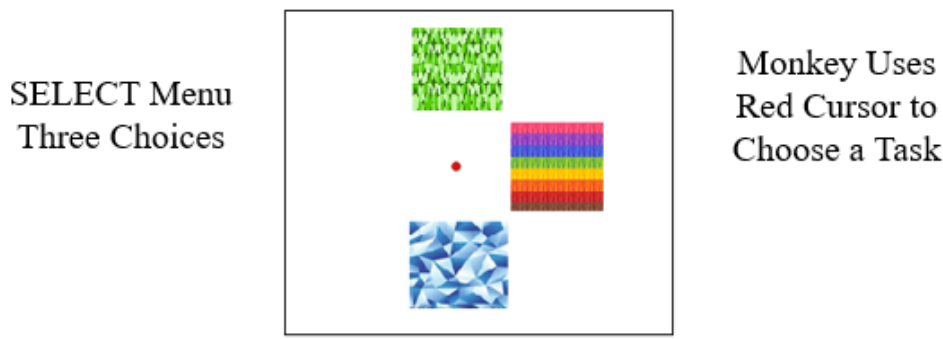

\section{If Monkey Chooses PURSUIT or PURSUIT Decoy}

Red Cursor Chases Green Target

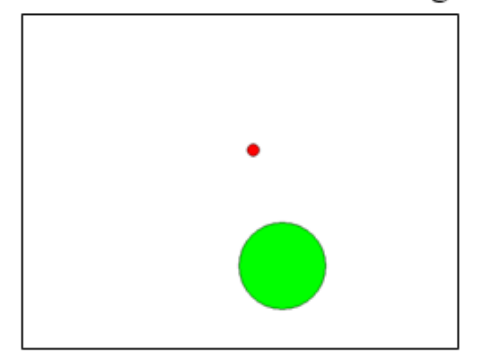

Cursor Remains Within Target

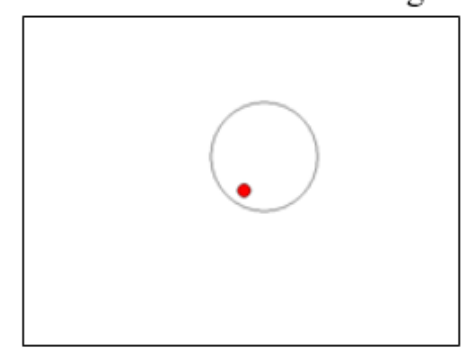

If Monkey Chooses MTS or MTS Decoy

Sample at Center, Matches at Top

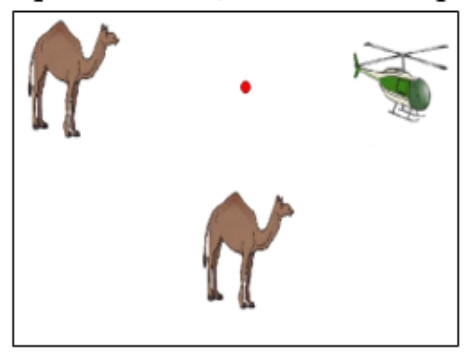

Monkey Selects Match

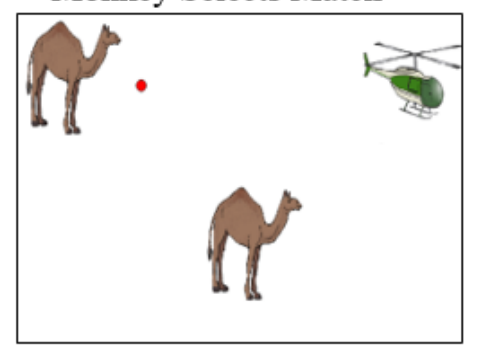

Figure 1. A schematic outline of the task. Monkeys first saw the panel at top, in which the SELECT menu offered them one, two, or three icons to choose from. In this example, the icon for PURSUIT is at bottom, the icon for MTS is at top, and the icon for decoy-PURSUIT is at right. If the monkey chose the icon for PURSUIT or decoy-PURSUIT, the task progressed as shown in the left column. If the monkey chose the icon for MTS or decoy-MTS, the task progressed as shown in the right column. At the end of each trial, the SELECT menu reappeared with the icons, which were randomly assigned to any of the four locations around the cursor's start location.

After a block of 50 choice trials (MTS vs. PURSUIT) was presented, the program assessed whether a monkey showed an extreme selection bias for the MTS task over the PURSUIT task or vice versa. If either icon was selected on more than $80 \%$ of the trials in this comparison, the program modified the task parameters for the next block. If parameters needed to be adjusted because of extreme preferences for the PURSUIT icon, we then increased how long contact had to be maintained inside the PURSUIT target to generate the 1-pellet food reward, as well as slowing the cursor in the decoyPURSUIT task. If parameters needed to be adjusted because of extreme preferences for the MTS icon, we slowed the cursor in the MTS task so that it took longer to complete those trials, and we also slowed the cursor equivalently in the decoy-MTS task. Monkeys advanced to the test phase in a session only when they completed a block of 50 MTS vs. PURSUIT trials without an extreme preference for one task over the other. If a monkey was still in the training phase when a session ended, the monkey continued at those parameters in the next session.

Test phase. The test phase consisted of 250 trials of each of the five trial types: MTS vs. decoyMTS, PURSUIT vs. decoy-PURSUIT, MTS vs. PURSUIT, MTS vs. PURSUIT vs. decoy-PURSUIT, and 
MTS vs. decoy-MTS vs. PURSUIT. These last two trial types were given to assess possible decoy effects. In these trials, three icons were presented including the MTS and PURSUIT icons and either the decoyMTS icon or the decoy-PURSUIT icon. Each of these five trial types was presented once in each block of five trials.

The experiment ended after 250 test trials of each type were completed. If a monkey did not complete these trials all in one session, the monkey was given another session the next day, or as soon as he was available again for testing.

\section{Results and Discussion}

One monkey, Luke, was discontinued in the experiment very early, because he would not engage in any of these tasks.

Training phase. Given the small sample size, we reported for each monkey the mean trial durations for the PURSUIT and PURSUIT decoy tasks. We also reported the percentage of trials correct for the MTS and MTS decoy tasks, and the total number of training trials completed with each task for each monkey. We also presented the overall choice percentages when two choices were given to the monkeys. Training data are in Table 1.

Table 1

Results from the Training Phase of Experiment 1 for each Monkey

\begin{tabular}{|c|c|c|c|c|c|c|c|c|}
\hline & \multicolumn{4}{|c|}{ MTS \% Correct } & \multicolumn{4}{|c|}{ PURSUIT Mean RT } \\
\hline & $\begin{array}{c}\text { Total } \\
N\end{array}$ & $\begin{array}{l}\text { MTS } \\
\text { Only }\end{array}$ & $\begin{array}{l}\text { MTS } \\
\text { Decoy }\end{array}$ & MTS-PUR & $\begin{array}{c}\text { Total } \\
N\end{array}$ & $\begin{array}{c}\text { PURSUIT } \\
\text { Only }\end{array}$ & $\begin{array}{l}\text { PURSUIT } \\
\text { Decoy }\end{array}$ & MTS-PUR \\
\hline Chewie & 57 & $100(9)$ & $100(9)$ & $94.9(39)$ & 29 & $3.05(9)$ & $8.66(9)$ & $3.66(11)$ \\
\hline Han & 38 & $66.7(9)$ & $55.6(9)$ & $50(20)$ & 46 & $8.45(8)$ & $8.39(8)$ & $8.18(30)$ \\
\hline Hank & 29 & $44.4(9)$ & $11.1(9)$ & $72.73(11)$ & 56 & $3.06(9)$ & $7.79(9)$ & $3.32(38)$ \\
\hline Lou & 109 & $95.3(43)$ & $90.5(42)$ & $87.5(24)$ & 308 & $5.05(42)$ & $13.29(41)$ & $5.87(225)$ \\
\hline Murph & 133 & $90(50)$ & $82(50)$ & $69.7(33)$ & 365 & $7.95(49)$ & $19.75(46)$ & $7.93(261)$ \\
\hline Obi & 40 & $100(9)$ & $66.7(9)$ & $72.27(22)$ & 46 & $2.90(9)$ & $19.14(9)$ & $4.53(28)$ \\
\hline
\end{tabular}

Note. The column headers refer to the trial types. In the "Only" and "Decoy" conditions, MTS, MTS decoy, PURSUIT, or PURSUIT decoy was the only icon available to choose. For the MTS-PUR trials, there were two choices onscreen and the monkey's choice on that trial led to either an MTS or PURSUIT trial. The numbers in parentheses are the trial counts for each of those specific trial types within the two tasks.

At the group level, there was no difference in the performance (\% correct) of the monkeys on the MTS task depending on whether the MTS task was performed when it was the only option offered $(M=$ $82.7 \%)$, whether it was chosen over the PURSUIT icon in binary choice sets $(M=75.3 \%)$, or whether it was presented in its decoy version $(M=67.6 \%), F(2,10)=1.56, p=.26, \eta_{\mathrm{p}}^{2}=.24$. This tells us that, once MTS had been selected as a task, it was equally challenging in all cases, which makes sense given that the only difference in both versions was how slowly the cursor moved to a match option. However, the responses times for those three conditions were significantly different, $F(2,10)=43.52, p<.001, \eta_{\mathrm{p}}{ }^{2}=$ .90. As expected, the monkeys took longer $(M=8.65 \mathrm{~s})$ to make a matching choice on the decoy version of the task than on the trials where only the MTS icon was available $(M=3.46 \mathrm{~s})$ or when the MTS task was chosen over PURSUIT $(M=3.86 \mathrm{~s})$.

Regarding the response times during training in capturing and maintaining the target in PURSUIT, there were differences in the performance of the monkeys depending on whether PURSUIT was the only icon presented $(M=2.55 \mathrm{~s})$, whether it was chosen over the MTS icon in the binary choice trial $(M=2.11 \mathrm{~s})$, or whether it was performed in its decoy version $(M=5.49 \mathrm{~s})$. These differences were statistically significant overall, $F(2,10)=11.20, p<.005, \eta_{\mathrm{p}}{ }^{2}=.69$. Planned comparisons indicated that trial latencies were significantly shorter for the PURSUIT task compared to the decoy version, both when PURSUIT was offered as the only choice, $t(5)=3.39, p=.019,95 \%$ CI $[1.76,12.76]$, and when 
PURSUIT was chosen over MTS, $t(5)=-3.33, p=.021,95 \%$ CI $[-13.76,-1.76]$. However, there was no difference in trial latencies between trials in which PURSUIT was presented solo or when presented with MTS, $t(5)=-1.81, p=.13,95 \%$ CI $[-1.22,0.21]$. This confirms that the decoy version of PURSUIT took significantly longer to complete than the normal version.

Overall, the training data indicated that the decoy tasks were functionally what we anticipated: inferior versions of each of the original tasks, where both tasks took longer to perform and thus should have been less preferred in terms of monkeys choosing to engage them. However, unlike in some past studies (Beran et al., 2007; Washburn et al., 1991), we did not find a performance advantage for trials in which the monkeys chose a task (MTS or PURSUIT) versus situations in which they were forced to do one of those same tasks on solo-task trials.

Test phase. These results for trials with decoy stimuli present are shown in Figure 2. The three graphs show the total trial counts for each icon that was selected (either the normal MTS icon, the normal PURSUIT icon, or either of the decoy icons). We assessed for each monkey the proportion of trials selecting each icon as a function of whether the decoy was for the MTS or PURSUIT task. We did this using a 3x2 Chi Square test for each monkey except for Han, for whom the low frequencies of choosing the decoy indicated that the Fisher's exact test was the appropriate test. The results are shown in Table 2. For only two monkeys was this test significant, indicating that whichever decoy was present did not appear to influence task choices. To assess choice behavior another way, we did not include choices of the decoy stimuli, to see if the relative frequencies of choosing MTS or PURSUIT differed depending on which decoy stimulus was present. They did not for any monkey except for Chewie, who showed an outcome that just exceeded statistical levels of significance.

We also compared the proportion choice for MTS and PURSUIT when they were in a trinary choice set compared to when they were in the binary choice set (no decoy present). The results are shown in Table 3. Two Chi Square tests were conducted in this way, one for trinary sets with the MTS decoy and another for trinary sets with the PURSUIT decoy. In the comparison with trinary sets with the MTS decoy, there were no significant differences in task choice across binary and trinary sets for any of the monkeys except for Hank and Murph. When PURSUIT decoy was in the trinary set, there were no significant differences in the task choices of any of the monkeys.

One strong and clear conclusion from Experiment 1 was that decoy effects were not present in the performances of these monkeys. When offered choices between tasks, the monkeys' relative preferences for one task over the other did not change when decoys were introduced that were inferior versions of either of those two tasks. In a minority of cases, for monkeys Chewie, Hank, and Murph, the statistical analysis suggested the presence of a weak decoy effect. Thus, the appropriately conservative conclusion was that decoy effects were not present in this experiment.

That said, when we examined these data, we realized that, despite our training regimen, the monkeys did not appear to consistently maintain relative levels of indifference or only slight preference for one task over the other during the test phase, and in some cases decoy icons were chosen more often than would have been expected (Figure 3). Post hoc to the formal analyses to look at the decoy effects, we found that there were extreme preferences for those binary test trials where only MTS and PURSUIT icons were offered as choices (Figure 3). Chewie and Han strongly preferred the MTS task and Hank strongly preferred the PURSUIT task. More concerning was that when MTS and the decoy MTS icons were presented, Murph selected MTS on only 70.3\% of trials, and Obi selected MTS on only 55.2\%. Lou chose PURSUIT over its decoy on only $73.2 \%$ of trials, and Chewie, Han, and Obi were basically indifferent between PURSUIT and its decoy version when those were the two options. This indicated to us that perhaps our training regimen, using $80 \%$ as a threshold, had not allowed the monkeys sufficient time and experience to reach a point where MTS and PURSUIT were fairly equivalent in terms of preferences, and were both preferred over their decoys. Thus, we replicated the experiment, using all new icons, and requiring a closer to equivalent preference level for MTS and PURSUIT before the test phase would begin. 
A)

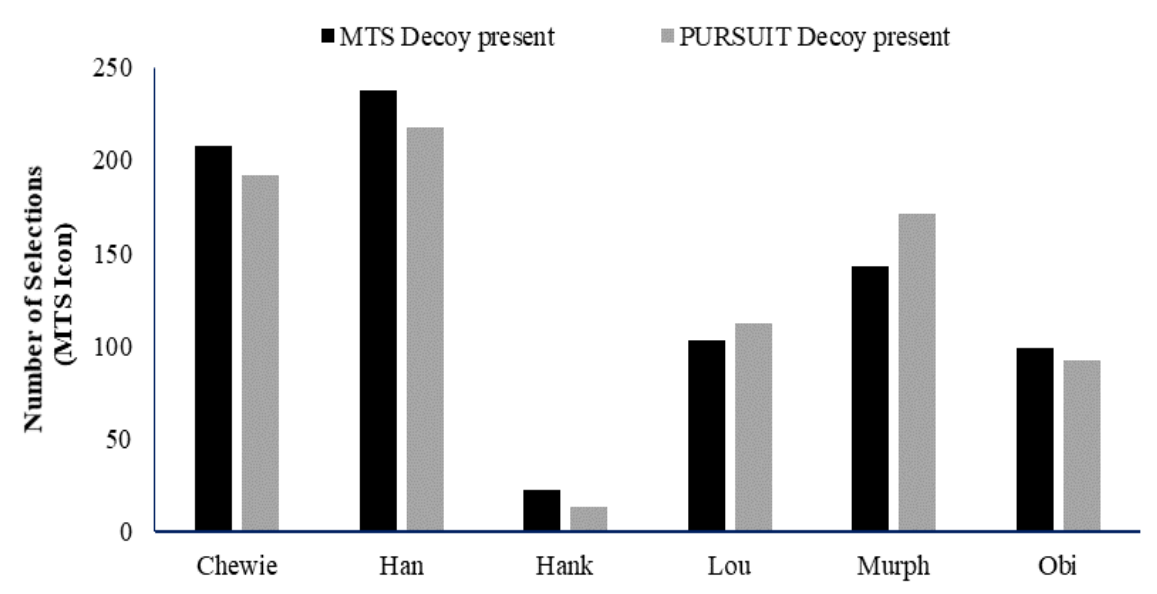

B)

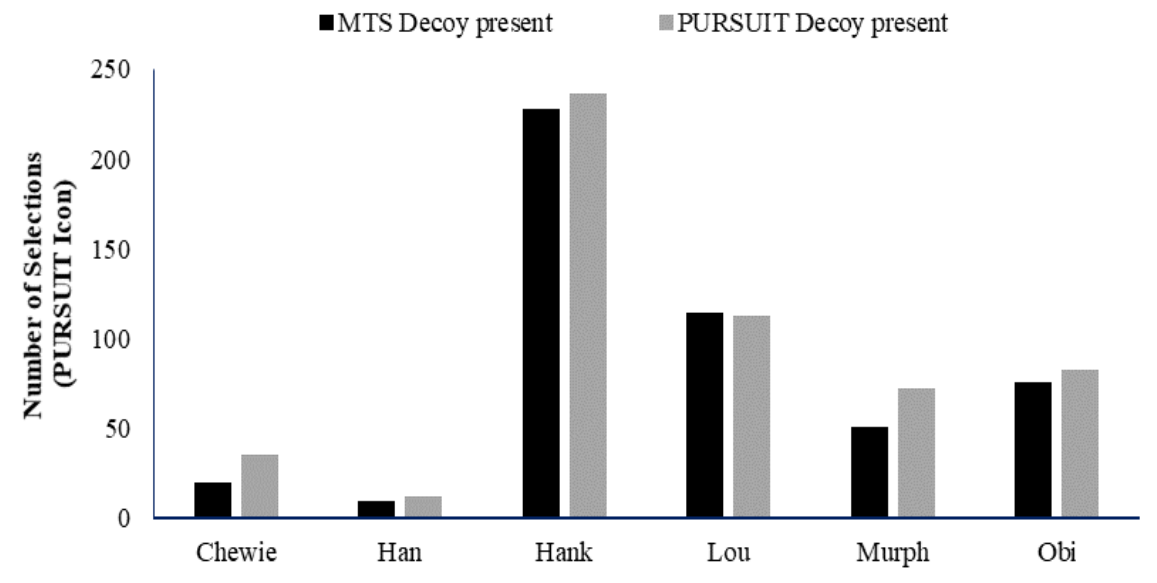

C)

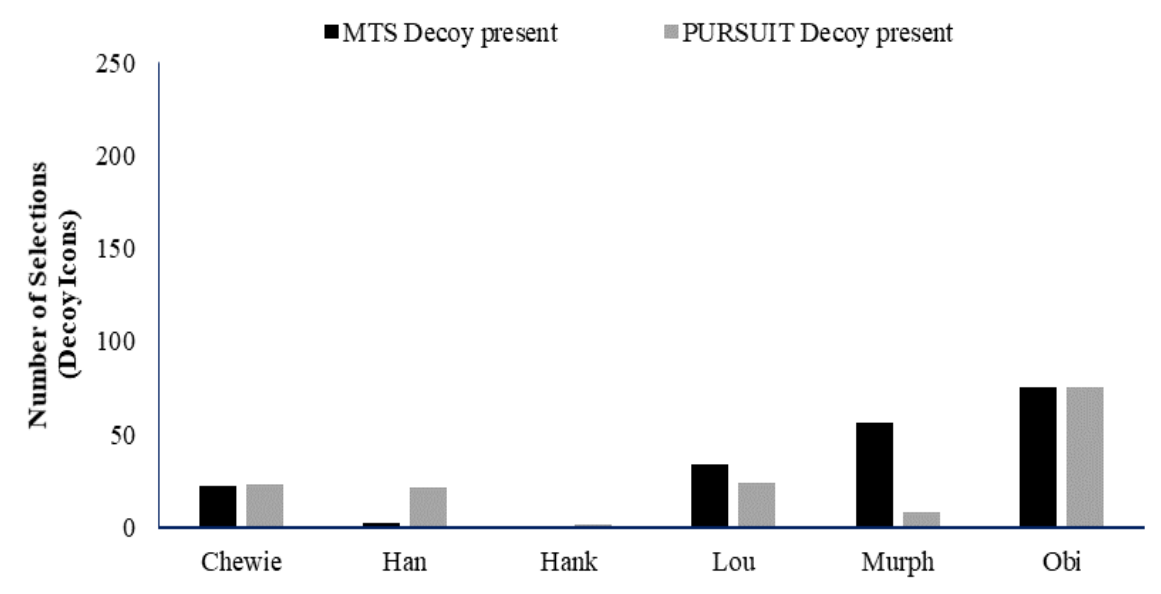

Figure 2. The results for trials with decoy stimuli present. A) shows the total trial counts for selection of the MTS icon, B) shows counts for selection of the PURSUIT icon, and C) shows counts for selection of either decoy icon. In each graph, the data are presented as a function of whether the MTS decoy was presented or the PURSUIT decoy was presented on that trial. 
Table 2

Results for Statistical Tests for Experiment 1 and Experiment 2 for each Monkey

\begin{tabular}{|c|c|c|c|c|c|c|c|c|}
\hline & \multicolumn{4}{|c|}{ Experiment 1} & \multicolumn{4}{|c|}{ Experiment 2} \\
\hline & $\begin{array}{c}\text { Chi Square } \\
(d f=2)\end{array}$ & $p$ & $\begin{array}{c}\text { Chi Square } \\
(d f=1)\end{array}$ & $p$ & $\begin{array}{c}\text { Chi Square } \\
(d f=2)\end{array}$ & $p$ & $\begin{array}{l}\text { Chi Square } \\
(d f=1)\end{array}$ & $p$ \\
\hline Chewie & 4.75 & .09 & 4.12 & .042 & 5.5 & .06 & 0.10 & .75 \\
\hline Han & 16.75 & .0002 & 0.16 & .68 & & Not $\mathrm{Te}$ & $d---$ & \\
\hline Hank & & NS* & 1.93 & .16 & 3.72 & .16 & 1.98 & .16 \\
\hline Lou & 2.1 & .35 & 0.15 & .69 & 5.65 & .06 & 0.01 & .92 \\
\hline Murph & 42.08 & $<.0001$ & 0.44 & .50 & & NS* & 4.96 & .03 \\
\hline Obi & 0.56 & .76 & 0,41 & .52 & 2.47 & .29 & 0.87 & .45 \\
\hline
\end{tabular}

Note. In each experiment, the first Chi Square value comes from each monkey's choice frequencies for the MTS, PURSUIT, or DECOY icons as a function of which decoy was presented on that trial. The second Chi Square value excludes choices of the decoy icons and only compared choices of MTS and PURSUIT icons as a function of which decoy was presented on that trial. * indicate Fisher's exact test.

Table 3

$\underline{\text { Results for Statistical Tests for Experiment } 1 \text { and Experiment } 2 \text { for each Monkey }}$

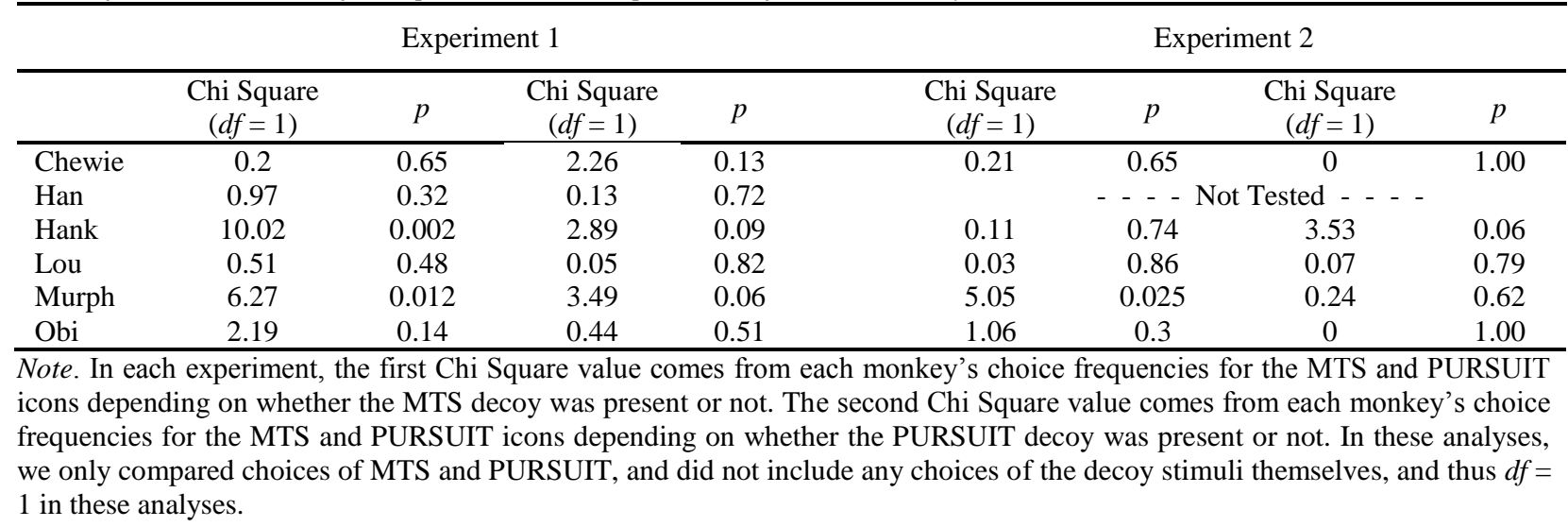


- MTS $=$ PURSUIT

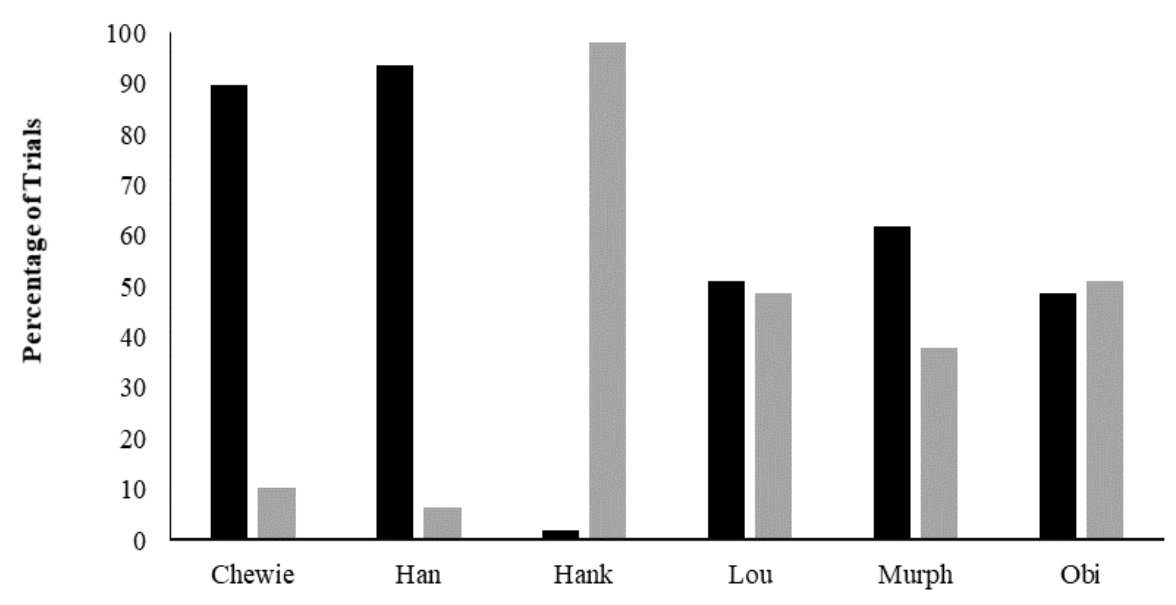

-MTS MTS-Decoy

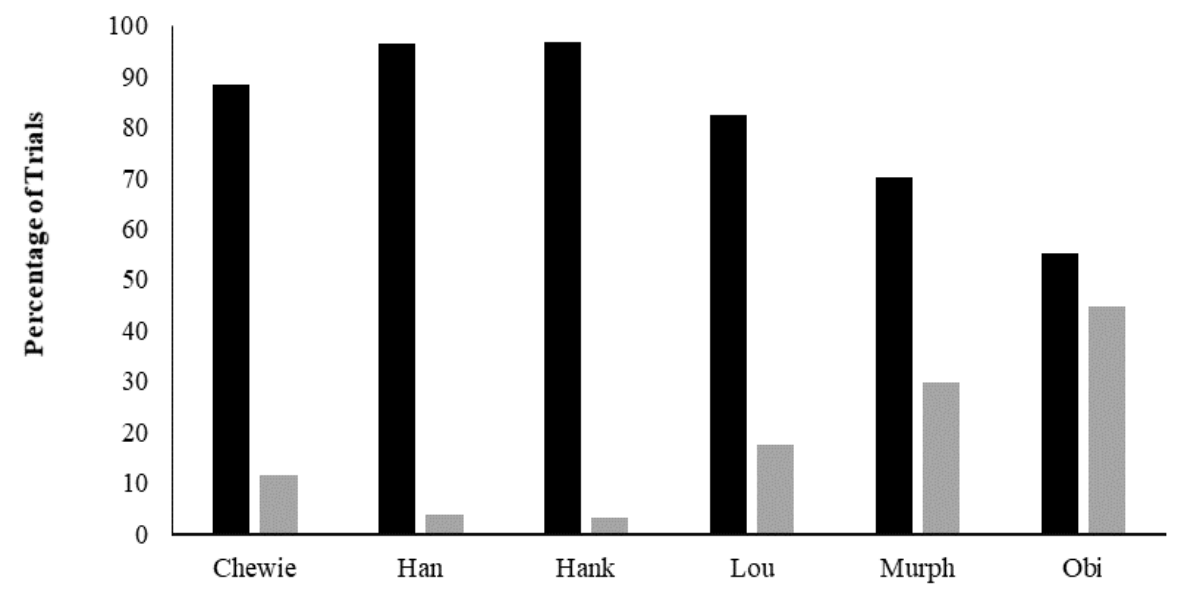

-PURSUIT $=$ PURSUIT-Decoy

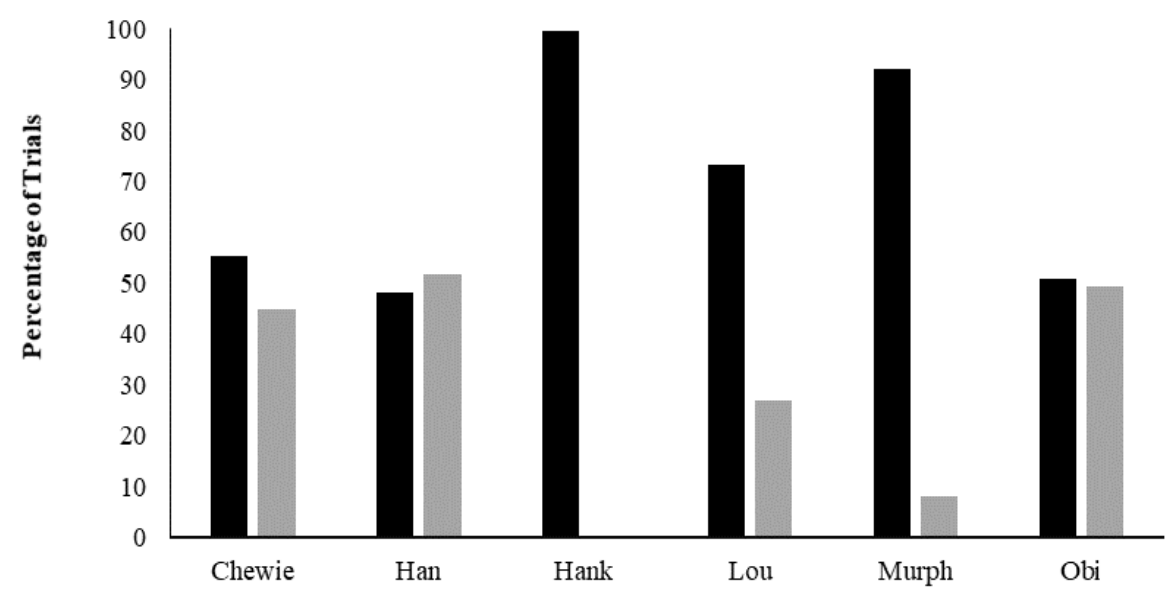

Figure 3. Monkeys' choices for each pair of icons presented in the test phase of Experiment 1. The paired bars indicate which two icons were presented. 


\section{Experiment $2^{1}$}

\section{Method}

Participants. We tested five of the six monkeys who completed Experiment 1. Monkey Han was not available because of assignment to an unrelated experiment.

Apparatus. This was the same as in Experiment 1. New icons were introduced for each task, including for each decoy version of each task.

Design and procedure. The design was the same as in Experiment 1. Monkeys first worked in the training phase and the same trial blocks were presented as in Experiment 1. Now, however, after each block of trials in which 50 choice trials (MTS vs. PURSUIT) was presented, the program assessed whether a monkey showed a selection bias in excess of $68 \%$ (rather than the $80 \%$ used in Experiment 1). If either icon was selected on more than $68 \%$ of the trials in this comparison, the program modified the task parameters for the next block, in the same manner as in Experiment 1.

The test phase again consisted of 250 trials each of five trial types: MTS vs. decoy-MTS, PURSUIT vs. decoy-PURSUIT, MTS vs. PURSUIT, MTS vs. PURSUIT vs. decoy-PURSUIT, and MTS vs. decoy-MTS vs. PURSUIT. The experiment again ended after 250 test trials of each type were completed. All other aspects of the experiment were the same as in Experiment 1.

\section{Results and Discussion}

Training phase. The mean trial durations for the PURSUIT and PURSUIT decoy tasks, the percentage of trials correct for the MTS and MTS decoy tasks, and the total number of training trials completed with each task for each monkey during training are shown in Table 4. At the group level, there was no difference in the performance (\% correct) of the monkeys on the MTS task depending on whether the normal MTS task was performed when it was the only option offered $(M=85.3 \%)$, whether it was chosen over the PURSUIT icon $(M=87.3 \%)$, or whether it was presented in its decoy version $(M=$ $81.9 \%), F(2,8)=1.01, p=.41, \eta_{\mathrm{p}}^{2}=.20$. However, the responses times for those three conditions were significantly different, $F(2,8)=9.34, p=.008, \eta_{\mathrm{p}}{ }^{2}=.70$. The monkeys took longer $(M=17.21 \mathrm{~s})$ to make a matching choice on the decoy version of the task than on the trials where only the normal MTS icon was available $(M=5.51 \mathrm{~s})$ or when the normal MTS task was chosen over PURSUIT $(M=5.14 \mathrm{~s})$.

Table 4

Results from the Training Phase of Experiment 2 for each Monkey

\begin{tabular}{|c|c|c|c|c|c|c|c|c|}
\hline & \multicolumn{4}{|c|}{ MTS \% Correct } & \multicolumn{4}{|c|}{ PURSUIT Mean RT } \\
\hline & $\begin{array}{c}\text { Total } \\
N\end{array}$ & $\begin{array}{l}\text { MTS } \\
\text { Only }\end{array}$ & $\begin{array}{c}\text { MTS } \\
\text { Decoy }\end{array}$ & MTS-PUR & $\begin{array}{c}\text { Total } \\
N\end{array}$ & $\begin{array}{l}\text { PURSUIT } \\
\text { Only }\end{array}$ & $\begin{array}{l}\text { PURSUIT } \\
\text { Decoy }\end{array}$ & MTS-PUR \\
\hline Chewie & 102 & $100(17)$ & $100(17)$ & $97.1(68)$ & 68 & $4.32(17)$ & $10.41(17)$ & $4.17(32)$ \\
\hline Hank & 193 & $43.3(67)$ & $52.2(67)$ & $59.3(59)$ & 473 & $7.38(67)$ & $18.25(65)$ & $7.06(341)$ \\
\hline Lou & 243 & $100(34)$ & $86.2(29)$ & $98.3(180)$ & 86 & $4.71(34)$ & $9.91(34)$ & $11.02(18)$ \\
\hline Murph & 238 & $94.1(34)$ & $82.1(28)$ & $96.0(176)$ & 90 & $3.41(34)$ & $8.89(34)$ & $4.10(22)$ \\
\hline Obi & 39 & $88.9(9)$ & $88.9(9)$ & $85.7(21)$ & 47 & $3.06(9)$ & $7.88(9)$ & $3.12(29)$ \\
\hline
\end{tabular}

Note. Data are presented here as in Table 1.

Regarding the response times during training in capturing and maintaining the target in PURSUIT, there were differences in the performance of the monkeys depending on whether PURSUIT was the only icon presented $(M=4.57 \mathrm{~s})$, whether it was chosen over the MTS icon $(M=5.89 \mathrm{~s})$, or whether it was performed in its decoy version $(M=11.07 \mathrm{~s})$. These differences were statistically

\footnotetext{
${ }^{1}$ This experiment was added after acceptance of the pre-registered manuscript. These methods were not included in the original pre-registered review process, but were reviewed by the editor for appropriateness with the original submission aims and proposed methodology.
} 
significant overall, $F(2,8)=10.56, p=.006, \eta_{\mathrm{p}}{ }^{2}=.73$. Planned comparisons indicated that trial latencies were significantly shorter for the normal PURSUIT task presented as the only icon compared to the decoy version, $t(4)=-5.83, p=.004,95 \%$ CI $[-9.58,-3.40]$. The same was true for the PURSUIT task chosen over MTS compared to PURSUIT decoy, although this difference approached conventional significance, $t(4)=-2.64, p=.058,95 \%$ CI [-10.63, 0.28]. However, there was no difference in the two PURSUIT outcomes depending on whether PURSUIT was the only icon offered, or when it was chosen over MTS when that pair of icons was presented, $t(4)=1.04, p=.36,95 \%$ CI $[-2.19,4.82]$. This confirms that the decoy version took significantly longer to complete than the normal version.

Overall, the training data again indicated that the decoy tasks were functionally what we anticipated: inferior versions of their matching tasks, where both tasks took longer to perform and thus should have been less preferred in terms of monkeys choosing to engage those tasks.

Test phase. The results for trials with decoy stimuli present are shown in Figure 4. The three graphs show the total trial counts for each icon that was selected (either the normal MTS icon, the normal PURSUIT icon, or either of the decoy icons). We again assessed for each monkey the proportion of trials selecting each icon as a function of whether the decoy was for the MTS or PURSUIT task. We did this using a 3x2 Chi Square test for each monkey except for Murph, for whom the low frequencies of choosing the decoy indicated that the Fisher's exact test was the appropriate test. The results are shown in Table 2. None of the monkeys showed a significant difference in the observed frequencies of these choices compared to the expected frequencies. To assess this differently, we again excluded choices of the decoy stimuli, to see if the relative frequencies of choosing MTS or PURSUIT differed depending on which decoy stimulus was present. They did not for any monkey except for Murph, who showed an outcome that just exceeded statistical levels of significance, and that largely did so because of his low frequency of choosing the MTS icon when the PURSUIT decoy was present.

We also again compared the proportion of choices for MTS and PURSUIT when they were in a trinary choice set compared to when they were in the binary choice set (no decoy present). The results are shown in Table 3. Two Chi Square tests were conducted in this way, one for trinary sets with the MTS decoy and another for trinary sets with the PURSUIT decoy. In the comparison with trinary sets with the MTS decoy, there were no significant differences in task choice across binary and trinary sets for any of the monkeys except for Murph. When PURSUIT decoy was in the trinary set, there were no significant differences in the task choices for any of the monkeys.

The strong and clear conclusion from Experiment 2 was that decoy effects again were not present in the performances of these monkeys. Again, in only one case, this time for monkey Murph, was there any analysis that reflected the presence of a decoy effect. Again, the appropriately conservative conclusion was that decoy effects were not present in this experiment.

In this experiment, the preferences for those trials where only MTS and PURSUIT icons were offered as choices were still extreme and differed between monkeys (Figure 5). Hank strongly preferred the MTS task and Murph and Lou strongly preferred the PURSUIT task. However, the monkeys now showed more appropriate preferences for MTS and PURSUIT over their respective decoy tasks. Only Obi showed less than a strong preference for MTS over decoy-MTS, and Obi and Chewie also showed a moderate preference for PURSUIT over decoy-PURSUIT. Thus, we felt confident that in this experiment we did introduce objectively inferior decoy tasks that the monkeys came to show consistently strong preferences against in nearly all cases. 
A)

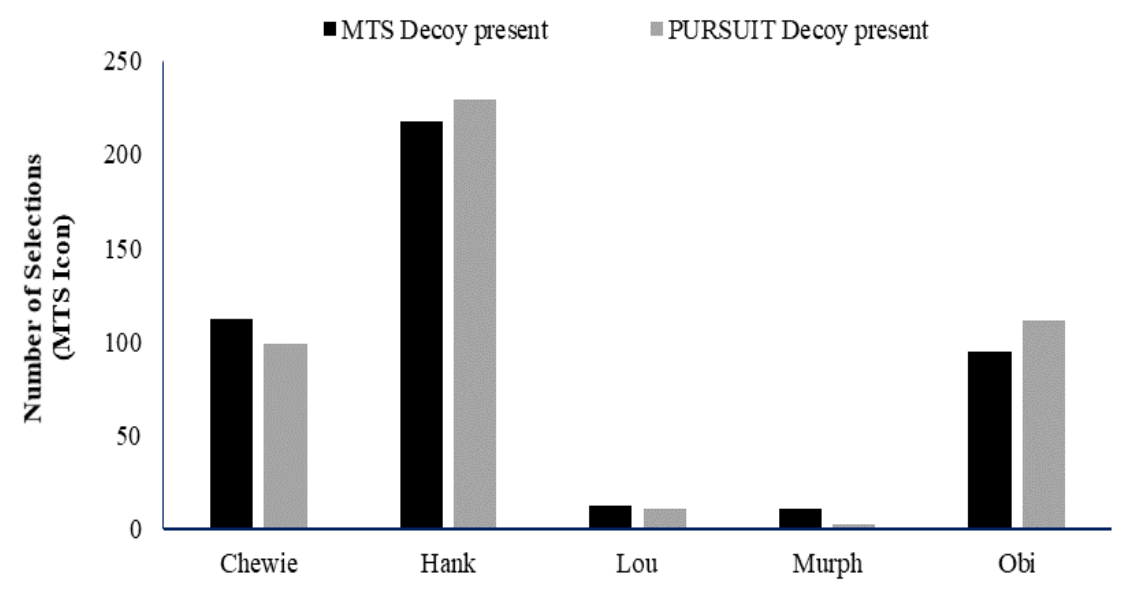

B)

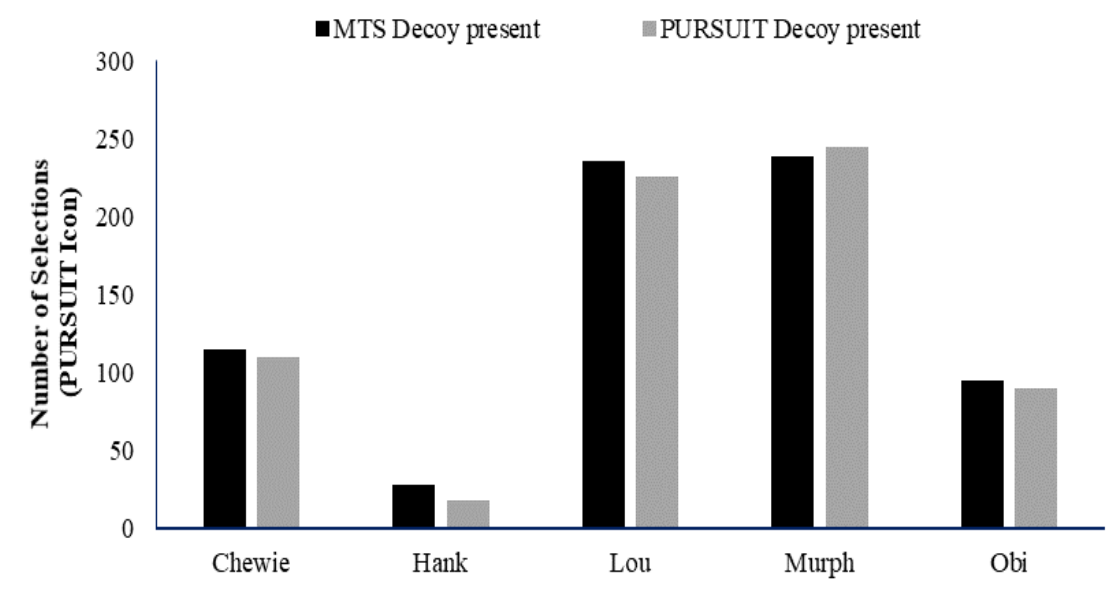

C)

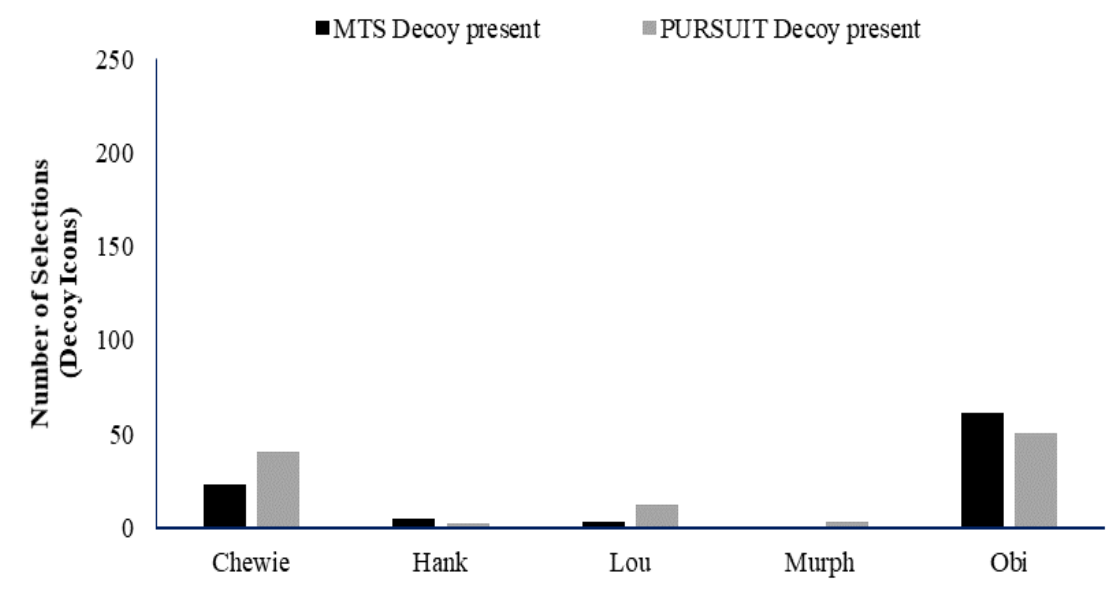

Figure 4. The results for trials with decoy stimuli present. A) shows the total trial counts for selection of the MTS icon, B) shows counts for selection of the PURSUIT icon, and C) shows counts for selection of either decoy icon. In each graph, the data are presented as a function of whether the MTS decoy was presented or the PURSUIT decoy was presented on that trial. 

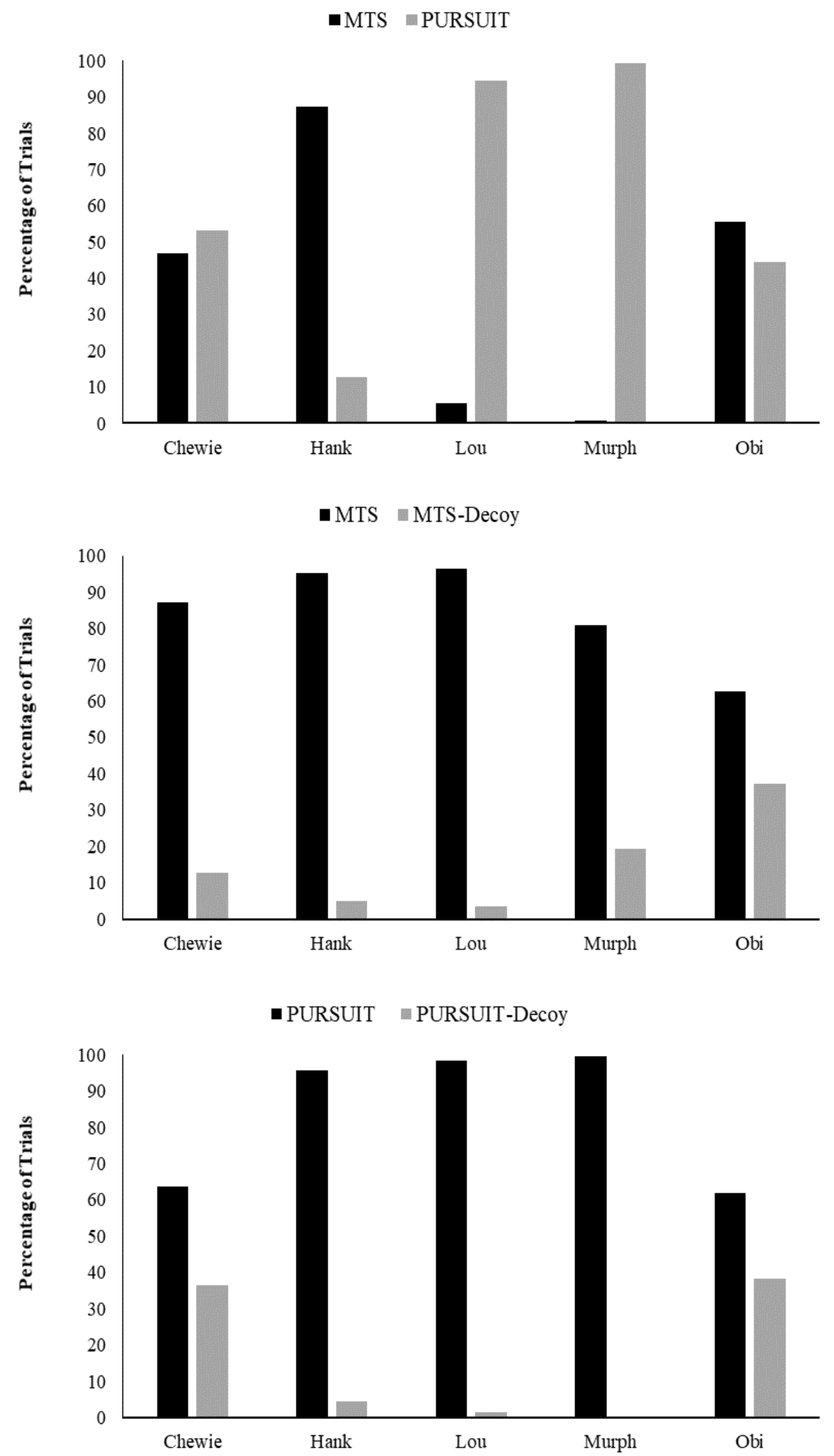

Figure 5. Monkeys' choices for each pair of icons presented in the test phase of Experiment 2. The paired bars indicate which two icons were presented. 


\section{General Discussion}

Rhesus monkeys did not show a decoy effect in the present study using a computerized taskselection procedure. Monkeys were presented with two tasks, including a psychomotor task (PURSUIT) and a conceptual task (MTS). Latency to trial completion was increased (via reduced cursor speed) to create the inferior decoy options for both tasks, effectively decreasing reinforcement rate for either decoy option. Monkeys performed very well across all tasks, including original tasks and the decoy versions of both tasks, learning to respond appropriately for each game. However, preference for one of the original two tasks did not shift when the third, inferior decoy versions were included in a choice set, except in the case of a small number of monkeys in each experiment whose choice behavior was affected in the anticipated manner by the decoy task. These results stand in contrast to our former research investigating perceptual decoy effects in this same species, and even within some of these same monkeys (Parrish et al., 2015) as well as comparative studies investigating the asymmetric dominance effect in other species (Bateson, 2002; Bateson et al., 2002; Scarpi, 2011; Shafir et al., 2002). A recent value-based decoy study with capuchin monkeys yielded a similar finding as reported here, in which relative preferences for one of two computerized icons associated with foods (Experiment 1) or one of two juice options with varying sugar concentrations (Experiment 2) were unaffected by the introduction of a third, inferior decoy option (Cohen \& Santos, 2017).

Several task differences between the current work and the previous perceptual decoy study (Parrish et al., 2015) emerged that may have led to the lack of decoy effect reported here. In the current work, the SELECT paradigm relied upon the monkeys' ability to associate symbolic icons with their appropriate tasks, which included unique icons for the original tasks as well as each decoy task. Also, the current task was designed under the assumption that each monkey would prefer the original tasks to equivalent degrees, as well as yield stable task preferences throughout testing. Additionally, 'preference' in the current task was not inherent to the stimuli themselves as is the case in food-based comparative paradigms and arguably even with our previous size-discrimination paradigm in which monkeys quickly and consistently learned to select the larger of two or three stimuli on screen. These task features differed from the relatively simpler perceptual paradigm in which monkeys engaged a size-discrimination procedure that included a non-symbolic decoy stimulus (i.e., a smaller rectangle that was congruent or incongruent in orientation with the largest rectangle) on probe trials. The motivation for the current design was to establish whether the decoy effect would emerge similarly in these same monkeys for a more complex preference task using symbolic stimuli, which is in line with the vast majority of research with human adults on the decoy effect using higher-order decisional tasks (e.g., Doyle et al., 1999; Huber et al., 1982; Pan et al., 1995; Pettibone \& Wedell, 2000; Sedikides et al., 1999; Zhang \& Zhang, 2007).

Because the decoy option typically operates to shift relative preference between two choices, exact equivalence between these original options should not be necessary for the asymmetric dominance effect to emerge. However, some monkeys exhibited strong biases for one of the original tasks across both experiments in the current study, strongly preferring MTS or PURSUIT when presented in a binary choice. These strong biases to choose one of the two original tasks may have prevented any decoy effect from emerging in the trinary choice set. Notably, these biases extended to Experiment 2 where we introduced a more conservative training criterion (E1 - 80\% vs. E2 - 68\%) to better ensure more equivalent preference between MTS and PURSUIT. Despite meeting criterion for training, most monkeys reverted to a strong bias for one of the two tasks in the test phase. This pattern of behavior may be attributed to the larger number of test trials $(1,250)$ compared to training trials (typically fewer than 200 trials per monkey). Additionally, the monkeys had a relatively long testing history with these specific tasks. Despite the training requirement to learn the association between novel icons and these tasks, the animals may have had pre-existing biases for one task over the other that negated our attempts to establish lasting equivalent preferences between tasks. Without this, a decoy effect may not be likely to emerge. As such, future studies may introduce new primary tasks that have no/little history for subjects or test for relative preference for the original tasks during or after testing to ensure equivalence.

The decoy effect, similar to many other forms of 'irrational' decision-making, should operate as a 
slight alteration to relatively fluid choice behavior. This is in fact consistent with our first study of this phenomenon in which monkeys exhibited a slight decoy effect in the first experiment of Parrish et al. (2015). Monkeys performed near ceiling when presented with two differently-sized rectangles in the binary choice set, which we predicted may have masked a stronger decoy effect from emerging. A more robust decoy effect emerged when we increased the difficulty such that the monkeys were no longer performing near ceiling in the binary choice set. This provided a greater window to observe the subtleties introduced by a decisional bias like the decoy. To use an example from a consumer decision-making standpoint, a customer who is extremely loyal to a particular brand should be less influenced by inferior or non-viable decoy alternatives introduced to a choice set as compared to a consumer who experiences relatively less brand-loyalty. The marketing literature discusses the role of initial preference in the binary choice set (along with a number of other variables) as a limitation to the decoy effect. The stronger the preference for one of the two original alternatives, the more negatively that strong preference impacts the effectiveness of the decoy option in shifting choice behavior (e.g., Mishra, Umesh, \& Stem, 1993). Future investigations of the decoy effect, particularly paradigms that assess preference behavior among animals, are needed to assess the role of initial preference between original options on decoy effectiveness in comparative studies. As previously discussed, the use of relatively novel tasks that the animals have had less experience with may lead to more equivalent and stable preference-patterns throughout testing.

Interestingly, monkeys selected the decoy option at higher than expected rates in the trinary choice sets comparing the two original tasks to either decoy task, as well as in the binary choice between the original task and its decoy. This was particularly evident in Experiment 1 . An elevated preference for the decoy option was unexpected and indicates that perhaps the monkeys did not associate the icons with the appropriate task. Selection of the decoy option was reduced in Experiment 2, in which monkeys experienced increased training that likely improved the association between task icon (novel in E2) and the given task. Although decoy paradigms that do not rely upon symbolic icons for preference testing can circumvent this issue, those that make use of symbolic choice options may need to be designed more carefully with regard to this issue of decoy attractiveness/differentiation from the original options.

A secondary objective in the current study was to assess how choice behavior can affect subsequent task performance with the prediction that chosen tasks would lead to elevated performance patterns relative to the same task that was instead assigned to the monkey. Performance was measured in terms of percent correct for MTS and trial latency for PURSUIT. We found no performance differences when monkeys chose to complete a task (in binary and trinary choice sets) versus when it was assigned to them (in forced Training trials). This finding was inconsistent with previous work using the SELECT battery with rhesus monkeys (Washburn et al., 1991) and capuchin monkeys (Perdue et al., 2014). However, unlike in those previous task batteries, the current task options were limited to relatively few choices (two tasks versus up to five tasks). In addition, we chose two tasks that were fairly easy in terms of their actual motor and cognitive requirements, and so this might explain why a performance advantage was not seen on those trials where MTS or PURSUIT was chosen versus when it was assigned.

We offer a few final thoughts on approaches to conceptual decoy effects with animals. First, although we designed our experiment to avoid extreme preferences (especially in Experiment 2) so that potential decoy effects might emerge, it is important to confirm whether such preferences "creep back" into any experiment that involves many choices and large numbers of trials. In hindsight, we could have created two new tasks rather than manipulate two familiar ones, and allowed the monkeys to progress in their experiences of those tasks to a point of near equivalence in terms of preference for either task, then, introduce the decoy stimuli, rather than giving monkeys both tasks in both versions from the beginning. Our concern was in giving them roughly equal experience with decoys and non-decoy options, and this is important, but perhaps first establishing that there is a consistent equivalence between the two focal tasks is more important. Second, we believe that decoy effects, when they are present, may be fairly limited and subtle, and so it is important to consider how best to create decoy stimuli that really do highlight the salient features of their partner tasks. Here, we used effort required for completing trials of the MTS and PURSUIT tasks as the means of doing that, but perhaps something like reward quality for each task and its decoy would be more effective. This might better align with previous, more naturalistic work on the 
decoy effect in some nonhuman animal species, and might therefore generate more positive evidence of an effect if one exists in the given setting. Third, however, is a recognition that perhaps there was no decoy effect in this study because comparisons among task types as presented here were not susceptible to such effects. At present, we can conclude that most of these monkeys did not seem to be affected by the introduction of clearly inferior task choices that shared properties in common with one of two otherwise distinctive tasks. Whether this reflects that no decoy effects occur, or whether it reflects a methodological limitation remains to be determined.

\section{Acknowledgements}

Support for this research was provided by NICHD (grant HD-060563). We thank the animal care and enrichment staff at the Language Research Center of Georgia State University for maintaining the health and wellbeing of the primates and making this research possible.

\section{References}

Bateson, M. (2002). Context-dependent foraging choices in risk-sensitive starlings. Animal Behaviour, 64, $251-260$. Bateson, M., Healy, S. D., \& Hurly, T. A. (2002). Irrational choices in hummingbird foraging behaviour. Animal Behaviour, 63, 587-596.

Beran, M. J., Klein, E. D., Evans, T. A., Antworth, R., \& Chan, B. (2007). Perceived control, motivation, and task performance in capuchin monkeys. In P. R. Zelick (Ed.), Issues in the psychology of motivation (pp. 171185). Hauppauge, NY: Nova Science Publishers.

Cohen, P. M., \& Santos, L. R. (2017). Capuchins (Cebus apella) fail to show an asymmetric dominance effect. Animal Cognition, 20, 331-345.

Doyle, J. R., O'Connor, D. J., Reynolds, G. M., \& Bottomley, P. A. (1999). The robustness of the asymmetrically dominated effect: Buying frames, phantom alternatives, and in-store purchases. Psychology \& Marketing, $16,225-243$.

Evans, T. A., Beran, M. J., Chan, B., Klein, E. D., \& Menzel, C. R. (2008). An efficient computerized testing method for the capuchin monkey (Cebus apella): Adaptation of the LRC-CTS to a socially housed nonhuman primate species. Behavior Research Methods, Instruments, \& Computers, 40, 590-596.

Herne, K. (1997). Decoy alternatives in policy choices: Asymmetric domination and compromise effects. European Journal of Political Economy, 13, 575-589.

Huber, J., Payne, J. W., \& Puto, C. (1982). Adding asymmetrically dominated alternatives: Violations of regularity and the similarity hypothesis. Journal of Consumer Research, 9, 90-98.

Huber, J., Payne, J. W., \& Puto, C. P. (2014). Let's be honest about the attraction effect. Journal of Marketing Research, 51, 520-525.

Luce, R. D. (1977). The choice axiom after twenty years. Journal of Mathematical Psychology, 15, $215-233$.

Mishra, S., Umesh, U., \& Stem, J. E. (1993). Antecedents of the attraction effect: An information-processing approach. Journal of Marketing Research, 30, 331-349.

Pan, Y., O'Curry, S., \& Pitts, R. (1995). The attraction effect and political choice in two elections. Journal of Consumer Psychology, 4, 85-101.

Parducci, A. (1965). Category judgment: A range-frequency model. Psychological Review, 72, 407-418.

Parrish, A. E., Evans, T. A., \& Beran, M. J. (2015). Rhesus macaques (Macaca mulatta) exhibit the decoy effect in a perceptual discrimination task. Attention, Perception, \& Psychophysics, 77, 1715-1725.

Perdue, B. M., Evans, T. A., Washburn, D. A., Rumbaugh, D. M., \& Beran, M. J. (2014). Do monkeys choose to choose? Learning \& Behavior, 42, 164-175.

Pettibone, J. C., \& Wedell, D. H. (2000). Examining models of nondominated decoy effects across judgment and choice. Organizational Behavior and Human Decision Processes, 81, 300-328.

Richardson, W. K., Washburn, D. A., Hopkins, W. D., Savage-Rumbaugh, S. E., \& Rumbaugh, D. M. (1990). The NASA/LRC computerized test system. Behavior Research Methods, Instruments, \& Computers, 22, $127-$ 131.

Scarpi, D. (2011). The impact of phantom decoys on choices in cats. Animal Cognition, 14, 127-136.

Sedikides, C., Ariely, D., \& Olsen, N. (1999). Contextual and procedural determinants of partner selection: Of asymmetric dominance and prominence. Social Cognition, 17, 118-139. 
Shafir, S., Waite, T. A., \& Smith, B. H. (2002). Context-dependent violations of rational choice in honeybees (Apis mellifera) and gray jays (Perisoreus canadensis). Behavioral Ecology and Sociobiology, 51, 180-187.

Trueblood, J. S., Brown, S. D., Heathcote, A., \& Busemeyer, J. R. (2013). Not just for consumers: Context effects are fundamental to decision making. Psychological Science, 24, 901-908.

Washburn, D. A., Hopkins, W. D., \& Rumbaugh, D. M. (1991). Perceived control in rhesus monkeys (Macaca mulatta): Enhanced video-task performance. Journal of Experimental Psychology: Animal Behavior Processes, 17, 123-129.

Wedell, D. H. (1991). Distinguishing among models of contextually induced preference reversals. Journal of Experimental Psychology: Learning, Memory, and Cognition, 17, 767-778.

Zhang, T., \& Zhang, D. (2007). Agent-based simulation of consumer purchase decision-making and the decoy effect. Journal of Business Research, 60, 912-922. 